\title{
Lenguaje oral y habilidades prelectoras en niños de 4 a 6 años. Un estudio sobre marginalidad y bilingüismo en el Perú
}

\author{
Oral language and pre-reading skills in children of four to six years. A study on \\ marginality and bilingualism in peru \\ Ricardo Canales G. ${ }^{1}$; Esther Velarde C.; Magali Meléndez J.; Susana Lingán H. \\ Universidad Nacional Mayor de San Marcos, Lima, Perú \\ (RECIBIDo 20/01/2014, ACEPTADo 25/05/2014)
}

\section{RESUMEN}

El estudio buscó determinar si la condición de marginalidad y el bilingüismo pueden estar afectando el desarrollo lingüístico oral y las habilidades prelectoras en niños de 4 a 6 años en tres zonas del Perú y, asimismo, establecer si el desarrollo de los componentes fonológicos, morfosintácticos y semánticos del lenguaje oral tiene relación con la conciencia fonológica, memoria verbal de corto plazo y otras habilidades prelectoras.

Para ello, se examinó a niños de educación inicial de zonas urbanas: Lima y Ate-Vitarte, y zonas rurales: monolingüe castellano en Cajamarca y bilingüe en Huancavelica, con el test breve de bilingüismo (Escobar 1978, González 2006), la Prueba ELO, de Ramos (2008), y la prueba BIL 3-6 (batería de inicio a la lectura), de Sellés (2008).

Los resultados mostraron diferencias significativas en el desarrollo lingüístico oral entre los grupos, a favor de niños de zonas urbanas. Los niños en condición de marginalidad rural- bilingüe, Huancavelica- Quintaojo, y en segundo término, los niños de CajamarcaHuayobamba, obtienen los más bajos rendimientos. Un resultado similar se aprecia en lo que respecta a las habilidades prelectoras.

Se halló correlación significativa entre el nivel de desarrollo de los diversos aspectos del lenguaje oral y las habilidades prelectoras.

Palabras clave: marginalidad, bilingüismo, lenguaje oral, conciencia fonológica, habilidades prelectoras

\section{ABSTRACT}

The purpose of the study was to determine if the condition of marginality and bilingualism could be affecting proper oral linguistic development and pre reading skills in children aged 4-6 years children of three areas of Perú, and also establish the correlation between the level of development of the components phonological, morph- syntactic and semantic oral language, phonological awareness, verbal short-term memory and other pre-reading skills.

1 Docente Auxiliar de la Facultad de Psicología de la UNMSM. Email: ricardo.is.canales@gmail.com 
For this purpose, urban children was examined children initial education of urban areas: Lima and Ate- Vitarte, and rural areas: Spanish monolingual: Cajamarca and bilingual: Huancavelica, with the short test bilingualism (Escobar 1978 , González 2006), Test ELO of Ramos (2008) and Test BIL 3-6 ( Battery start reading ) of Selles (2008) .

The results showed significant differences in oral language development between groups for urban children. Children in bilingual status - rural marginality: Huancaveliv: Quintaojo, and secondly, children: Cajamrca: Huayobamba, obtained lower yields. A similar result is seen with respect to the pre- reading skills.

Significant correlation was found between the levels of development between the various aspects of oral language with pre reading skills.

Keywords:

\section{INTRODUCCIÓN}

Una de las áreas más importantes de la investigación psicolinguística contemporánea es la de la adquisición del lenguaje (Berko y Berstein, 1999). Dicho abordaje científico, que fue asumido por psicólogos y psicolingüistas de renombre como Skinner, 1954; Piaget, 1926; Vigotski, 1964; Chomksy, 1954 y Bruner, 1986, trata de dar cuenta de cómo es que ocurre el proceso de estructuración de la maquinaria psicolinguística mental que finalmente le permitirá al niño el uso y manejo de aquella extraordinaria herramienta comunicacional y representacional, que viene a ser el lenguaje humano.

En nuestro medio, dicho estudio, es decir, explorar la adquisición y el desarrollo del lenguaje en el niño, tiene sus complejidades. Ello porque el nuestro es muy diverso en lo geográfico, lo cultural, lo socioeconómico y lo lingüístico. Aparte de la presencia de los llamados factores disortogénicos que influyen directamente en el desarrollo psicológico de los niños: pobreza extrema, marginalidad, analfabetismo de los padres, etc., entra a tallar el grave problema lingüístico. En el Perú, según Pozzi- Escot (1998), unos cinco millones de habitantes hablan el quechua, medio millón son hablantes del aymara y unos cuatrocientos mil peruanos manejan cuarenta y un códigos linguísticos amazónicos.

El tema de la adquisición del lenguaje ha sido estudiado con amplitud al interior de la psicología y de la psicolingüística. Al respecto, trascendiendo los afrontes asociacionistas (Skinner) y el innatismo chomskiano, en los últimos tiempos surgió el modelo transaccional de Bruner (partiendo de tesis de Vigotski e integrándolo al moderno enfoque cognitivo).

Lo esencial del enfoque bruneriano reside en señalar que, más allá del necesario dispositivo innato para la adquisición del lenguaje( L.A.D.), se hace imprescindible que el niño cuente con un sistema de apoyo microsocial (L.A.A.S.), en donde la madre juega un rol fundamental. En su proceso de desarrollo del lenguaje, el niño 
pasa por tres etapas: etapa de interacciones lingüísticas prelocutivas, que va de los 0 a los 12 meses; etapa de interacciones lingüísticas transaccionales, que va de los 12 a los 24 meses; y etapa de interacciones lingüísticas intencionales, que va de los 24 a los 36 meses. Se entiende que al finalizar todo ello, el niño habrá constituido su maquinaria psicolingüística mental, correspondiente a su lengua materna, con su componente fonológico, morfosintáctico, semántico y pragmático del lenguaje.

En nuestro medio, González (2006) ha sido uno de los pocos psicólogos que se abocó al estudio del proceso de adquisición del lenguaje oral. En "Exploración del desarrollo del lenguaje del niño peruano menor de tres años: un modelo interactivo", se planteó como objetivo central estudiar el nivel de frecuencia y calidad de interacciones lingüísticas producidas entre la madre y el niño en los primeros tiempos de vida, precisamente en la época en que se da la adquisición del lenguaje, en cuatro zonas del país: Lima, Vitarte, Cajamarca y Andahuaylas. Los resultados fueron que Andahuaylas presentaba un menor número de interacciones lingüísticas: 135, luego seguía Cajamarca: 175, a continuación Vitarte: 199, y Lima- Cercado: 238. Esto indicaba que el factor regional y de marginalidad influía directamente en dichos resultados, es decir, afectaba el desarrollo lingüístico de los niños del Perú, especialmente en las interacciones del primer tipo: prelocutivas (Bruner, op. cit.). Una de sus conclusiones fundamentales fue que el factor de MARGINALIDAD, se entrecruza directamente afectando el proceso de interacción verbal, tanto en lo cuantitativo como en lo cualitativo. Es decir, los niños se verán más afectados mientras más marginal sea su zona de pertenencia (rural y quechua hablante). Dicha interacción se verá afectada específicamente por el factor del BILINGÜISMO (que en nuestro país tiene carácter sustractivo), es decir, la interferencia en el habla de la madre de componentes de su lengua originaria, el quechua, a partir de lo cual interacciona con el niño.

Sobre las habilidades prelectoras, destaca la conciencia fonológica. Ella tiene que ver con la capacidad de manipular y reflexionar sobre los aspectos sonoros del lenguaje hablado. Existen diferentes niveles de conciencia fonológica, dependiendo del tipo de unidad lingüística sobre el cual el niño aplica la reflexión y la manipulación de los sonidos del habla. Estos niveles son: rimas, sílabas, intrasílabas y fonemas.

Respecto a la habilidad del conocimiento de letras, se ha demostrado que el número de letras que conoce el niño(a) presenta un alto grado de predictividad sobre el aprendizaje de la lectura. Este grado de predictividad aumenta si el conocimiento de la letra va asociado con la identificación fonológica. Por último, respecto al dominio semántico y sintáctico del lenguaje oral, se le entiende como la capacidad de realizar una emisión verbal usando los pronombres y palabras funcionales solicitados y, por otro lado, si es capaz de emitir un mensaje que expresa una respuesta esencial o categorial ante determinado vocabulario.

El problema respecto al déficit en el desarrollo psicolinguístico de los niños peruanos, como se aprecia, se hace mayor cuando se analizan las repercusiones en el campo educativo y, en particular, en el proceso del aprendizaje de la lectura y escritura. El $45 \%$ de los niños de $1^{\circ}$ grado en la sierra y el $45 \%$ de niños de la 
selva son repitentes o desertores escolares. El atraso escolar es también diferente en relación con la lengua nativa: los hispanohablantes se atrasan en 2 años, mientras los que tienen como lengua materna el quechua $u$ otra lengua nativa se atrasan 4 años.

Un estudio reciente (Canales, 2012) halló que a nivel de conciencia fonémica, conocimiento del nombre y sonido de las letras, así como en tareas que evalúan la memoria verbal, los aspectos sintácticos y semánticos del lenguaje, los bilingües incipientes rendían más bajo que los niños monolingües en castellano y bilingües avanzados.

Precisamente, siguiendo dicha línea de investigación, es que en el presente estudio se trató de ver qué es lo que pasaba con dicho desarrollo lingüístico en los años siguientes -según lo que estudió González-, es decir a los 4, 5 y 6 años, tomando como base los mismos grupos examinados: niños de área urbana: Lima- Cercado y Vitarte- Huaycán, y niños de área rural: Huancavelica (Quintaojos) y Cajamarca (Huayobamba).

En particular, se consideró importante examinar en una muestra de niños de diversas zonas del país, de qué modo los factores socioculturales -marginalidad (urbana-rural)- y la condición lingüística -bilingüe, monolingüe- afectarían su proceso de adquisición y desarrollo del lenguaje temprano, así como la formación de las habilidades prelectoras.

De ahí que para el presente estudio se diferenciaron cuatro grupos: Marginalidad nivel 1: Lima-Cercado; Marginalidad nivel 2: zona urbano-marginal: VitarteHuaycán; Marginalidad nivel 3: zona rural pero monolingüe castellano: Huayobamba; y Marginalidad nivel 4: Huancavelica-Quintaojos (zona rural y bilingüe, por la presencia del quechua).

De modo específico, queríamos ver qué nivel de desarrollo alcanzan sus procesos perceptivos fonológicos, morfosintácticos y semánticos del lenguaje oral, y la conciencia fonológica y habilidades prelectoras en niños de 4 a 6 años pertenecientes a distintas zonas del país con distintas condiciones de marginalidad.

\section{Objetivos}

1. Evaluar y diferenciar los grupos por niveles de marginalidad: nivel 1: LimaCercado, nivel 2: urbano-marginal, nivel 3: rural- marginal y nivel 4: rural y bilingüe.

2. Examinar sus niveles de desarrollo en adquisición del lenguaje oral: componentes fonológicos, morfosintácticos y semánticos.

3. Examinar las habilidades prelectoras: conciencia fonológica, memoria verbal y procesos de alto nivel preparatorios para la lectura.

4. Determinar si existen diferencias en dichos componentes y habilidades psicolingüísticas entre los niños de los grupos examinados. 
5. Determinar la relación existente entre el nivel de desarrollo de dichos componentes y habilidades que son prerrequisito para el aprendizaje de la lectura.

\section{Hipótesis}

Existen diferencias en el nivel de desarrollo de los componentes fonológicos, morfosintácticos y semánticos del lenguaje oral en los niños de la muestra en función de su condición de marginalidad.

1. Existen diferencias en el nivel de desarrollo de las habilidades prelectoras en los niños de la muestra en función de su condición de marginalidad.

2. Existe relación entre el nivel de desarrollo de los aspectos fonológicos, memoria verbal, aspectos morfosintácticos y semánticos del lenguaje oral y la conciencia fonológica, conocimiento alfabético y demás habilidades prelectoras en los niños de la muestra.

\section{MÉTODO}

El presente fue un estudio descriptivo, de tipo causal comparativo, en donde, diferenciando a los niños por condición de marginalidad, se trató de ver la influencia que pueden tener factores socioculturales y de bilingüismo sobre el desarrollo del lenguaje oral y de las habilidades prelectoras.

Metodológicamente, se tomó como variable independiente la pertenencia o no a uno de los 4 grupos y como variables asociadas al lenguaje oral y las habilidades prelectoras. Las variables de control fueron la edad, el grado escolar y la condición lingüística.

Al final se trató de establecer las diferencias entre los 4 grupos a nivel de los componentes del lenguaje y las habilidades prelectoras.

La población estuvo constituida por niños de 4 a 6 años de zonas del nivel 1: LimaCercado, 21 niños de la Institución Educativa "Ricardo Bentín"; nivel 2: urbanomarginal: 30 niños de I. E. 207 (Vitarte- Huaycán); nivel 3: rural- marginal: 31 niños de I.E. 230 (Huancavelica- Huayobamba); y nivel 4: rural y bilingüe: 18 niños (I. E. Huancavelica- Quintaojos). Total: 100 niños.

Se emplearon los siguientes instrumentos:

Test breve de bilingüismo quechua-castellano, de Escobar, en adaptación de González (2008).

Prueba BIL 3-6 (Batería de inicio a la lectura), de Sellés et al. (2008).

Prueba ELO: Evaluación del lenguaje oral, de Ramos, Cuadrado y Fernández (2008). 
A nivel procedimental, luego de haber seleccionado la muestra se procedió a la aplicación de los instrumentos de evaluación sobre bilingüismo para diferenciar los grupos y luego se aplicaron los tests de evaluación del lenguaje oral y de habilidades prelectoras, tanto en Lima como en provincias.

\section{RESULTADOS}

\section{Hipótesis 1}

Existen diferencias en el nivel de desarrollo de los componentes fonológicos, morfosintácticos y semánticos del lenguaje oral en los niños de la muestra en función de su condición de marginalidad.

Para el análisis comparativo del lenguaje oral y las habilidades prelectoras en función del nivel de marginalidad se ha utilizado la prueba, no paramétrica, Kruskall Wallis. Ello por tratarse de la comparación de puntuaciones ordinales entre cuatro grupos independientes.

Adicionalmente, en aquellas comparaciones en donde el resultado fue significativo se han realizado comparaciones por pares con la prueba $U$ de Mann Whitney, utilizando la corrección de Bonferroni para seis posibles pares.

En la tabla 1 se puede apreciar que existen diferencias significativas en seis de las ocho tareas que conforman la prueba que evalúa el lenguaje oral. Estas son: discriminación auditiva de fonemas, memoria verbal de oraciones, composición de oraciones, descripción de acciones, vocabulario I, comprensión oral de narraciones y en la puntuación total de la prueba.

Tabla 1. Comparación de las puntuaciones obtenidas en las tareas que evalúan el lenguaje oral, según grupos de marginalidad

\begin{tabular}{|c|c|c|c|c|c|c|}
\hline \multirow{3}{*}{$\begin{array}{c}\text { Tareas del } \\
\text { lenguaje oral }\end{array}$} & \multirow{3}{*}{$\begin{array}{l}\text { Sig. } \\
\text { Kruskall } \\
\text { Wallis }\end{array}$} & \multicolumn{4}{|c|}{ Puntuaciones medias obtenidas por los grupos } & \multirow{3}{*}{$\begin{array}{l}\text { U de Mann } \\
\text { Whitney con } \\
\text { corrección de } \\
\text { Bonferroni }\end{array}$} \\
\hline & & \multicolumn{4}{|c|}{ Mediana (rango) } & \\
\hline & & $\begin{array}{c}\text { Nivel } 1 \\
\text { Urbano- } \\
\text { monolingüe }\end{array}$ & $\begin{array}{l}\text { Nivel } 2 \\
\text { Urbano- } \\
\text { marginal }\end{array}$ & $\begin{array}{c}\text { Nivel } 3 \\
\text { Rural- } \\
\text { monolingüe }\end{array}$ & $\begin{array}{c}\text { Nivel } 4 \\
\text { Rural- } \\
\text { bilingüe }\end{array}$ & \\
\hline $\begin{array}{l}\text { Discriminación } \\
\text { auditiva de } \\
\text { fonemas }\end{array}$ & .036 & $6(0-12)$ & $8(3-9)$ & $6(0-12)$ & $6(4-10)$ & Nivel $2>$ Nivel 4 \\
\hline $\begin{array}{l}\text { Aspectos } \\
\text { fonológicos }\end{array}$ & .114 & $37(6-45)$ & $34(4-41)$ & $32(8-45)$ & $\begin{array}{c}33(18- \\
38)\end{array}$ & - \\
\hline $\begin{array}{l}\text { Memoria verbal } \\
\text { de oraciones }\end{array}$ & .000 & $4(0-10)$ & $4(0-10)$ & $4(0-8)$ & $50(0-6)$ & $\begin{array}{l}\text { Nivel } 1>\text { Nivel } 4 \\
\text { Nivel } 2>\text { Nivel } 4 \\
\text { Nivel } 3>\text { Nivel } 4\end{array}$ \\
\hline $\begin{array}{l}\text { Composición } \\
\text { de oraciones }\end{array}$ & .001 & $3(0-10)$ & $2(0-10)$ & $1(0-8)$ & $0(0-2)$ & $\begin{array}{l}\text { Nivel } 1>\text { Nivel } 4 \\
\text { Nivel } 2>\text { Nivel } 4 \\
\text { Nivel } 3>\text { Nivel } 4\end{array}$ \\
\hline
\end{tabular}


Ricardo Canales G.; Esther Velarde C.; Magali Meléndez J.; Susana Lingán H.

\begin{tabular}{|c|c|c|c|c|c|c|}
\hline $\begin{array}{l}\text { Descripción de } \\
\text { acciones }\end{array}$ & .000 & $8(0-10)$ & $8(0-10)$ & $6(0-10)$ & $3(0-7)$ & $\begin{array}{l}\text { Nivel } 1>\text { Nivel } 4 \\
\text { Nivel } 2>\text { Nivel } 4 \\
\text { Nivel } 3>\text { Nivel } 4\end{array}$ \\
\hline $\begin{array}{l}\text { Vocabulario I. } \\
\text { Señalar dibujos }\end{array}$ & .000 & $4(0-5)$ & $3(1-4)$ & $2(0-4)$ & $2(0-5)$ & $\begin{array}{l}\text { Nivel } 1>\text { Nivel } 3 \\
\text { Nivel } 1>\text { Nivel } 4 \\
\text { Nivel } 2>\text { Nivel } 4 \\
\end{array}$ \\
\hline $\begin{array}{c}\text { Vocabulario } \\
\text { II. Expresar } \\
\text { significado }\end{array}$ & .121 & $1(0-7)$ & $2(0-7)$ & $2(0.5)$ & $0(0-6)$ & - \\
\hline $\begin{array}{c}\text { Comprensión } \\
\text { oral de } \\
\text { narraciones }\end{array}$ & .005 & $2(0-3)$ & $2(0-5)$ & $1(0-2)$ & $0(0-3)$ & $\begin{array}{l}\text { Nivel } 1>\text { Nivel } 4 \\
\text { Nivel } 2>\text { Nivel } 4\end{array}$ \\
\hline Prueba total & .001 & $69(12-81)$ & $\begin{array}{c}59(18- \\
98)\end{array}$ & $56(20-82)$ & $\begin{array}{c}46(30- \\
66)\end{array}$ & $\begin{array}{l}\text { Nivel } 1>\text { Nivel } 4 \\
\text { Nivel } 2>\text { Nivel } 4\end{array}$ \\
\hline
\end{tabular}

Es importante señalar que en todas las tareas en las que existen diferencias significativas, el grupo que obtiene menores puntuaciones es el grupo 4, es decir, el conformado por niños bilingües provenientes de zonas rurales. Asimismo, se evidencia que no existen diferencias entre los grupos 1 y 2 en ninguna de las tareas.

\section{Hipótesis 2}

Existen diferencias en el nivel de desarrollo de las habilidades prelectoras en los niños de la muestra en función de su condición de marginalidad.

En la tabla 2 se aprecia la comparación realizada de las puntuaciones obtenidas en las tareas que evalúan habilidades prelectoras según grupo de marginalidad. Vemos que existan diferencias significativas en todas las tareas. Estas son: conocimiento alfabético, reconocimiento de palabras, conteo de palabras, aislar sílabas y fonemas, detección de rimas, conteo de sílabas, omisión de sílabas y en la puntuación total de la prueba.

Tabla 2. Comparación de las puntuaciones obtenidas en las tareas que evalúan las habilidades prelectoras, según grupos de marginalidad

\begin{tabular}{|c|c|c|c|c|c|c|}
\hline \multirow{3}{*}{$\begin{array}{c}\text { Tareas del } \\
\text { lenguaje oral }\end{array}$} & \multirow{3}{*}{$\begin{array}{l}\text { Sig. } \\
\text { Kruskall } \\
\text { Wallis }\end{array}$} & \multicolumn{4}{|c|}{ Puntuaciones medias obtenidas por los grupos } & \multirow{3}{*}{$\begin{array}{l}\text { U de Mann Whitney } \\
\text { con corrección de } \\
\text { Bonferroni }\end{array}$} \\
\hline & & \multicolumn{4}{|c|}{ Mediana (rango) } & \\
\hline & & $\begin{array}{c}\text { Nivel } 1 \\
\text { Urbano- } \\
\text { monolingüe }\end{array}$ & $\begin{array}{l}\text { Nivel } 2 \\
\text { Urbano- } \\
\text { marginal }\end{array}$ & $\begin{array}{c}\text { Nivel } 3 \\
\text { Rural- } \\
\text { monolingüe }\end{array}$ & $\begin{array}{c}\text { Nivel } 4 \\
\text { Rural- } \\
\text { bilingüe }\end{array}$ & \\
\hline $\begin{array}{c}\text { Conocimiento } \\
\text { alfabético }\end{array}$ & .000 & $6(0-19)$ & $6(0-20)$ & $1(0-13)$ & $1(0-7)$ & $\begin{array}{l}\text { Nivel } 1>\text { Nivel } 3 \\
\text { Nivel } 1>\text { Nivel } 4 \\
\text { Nivel } 2>\text { Nivel } 3 \\
\text { Nivel } 2>\text { Nivel } 4\end{array}$ \\
\hline
\end{tabular}


LENGUAJE ORAL Y HABILIDADES PRE LECTORAS EN NIÑOS DE 4 A 6 AÑOS. UN ESTUDIO SOBRE MARGINALIDAD Y BILINGÜISMO EN EL PERÚ

\begin{tabular}{|c|c|c|c|c|c|c|}
\hline $\begin{array}{l}\text { Reconocimiento } \\
\text { de palabras }\end{array}$ & .000 & $6(2-9)$ & $7(5-10)$ & $6(1-16)$ & $4(0-6)$ & $\begin{array}{l}\text { Nivel } 1>\text { Nivel } 2 \\
\text { Nivel } 1>\text { Nivel } 4 \\
\text { Nivel } 2>\text { Nivel } 3 \\
\text { Nivel } 2>\text { Nivel } 4 \\
\text { Nivel } 3>\text { Nivel } 4\end{array}$ \\
\hline $\begin{array}{l}\text { Conteo de } \\
\text { palabras }\end{array}$ & .000 & $3(0-6)$ & $3(0-6)$ & $2(0-5)$ & $0(0-2)$ & $\begin{array}{l}\text { Nivel } 1>\text { Nivel } 4 \\
\text { Nivel } 2>\text { Nivel } 4 \\
\text { Nivel } 3>\text { Nivel } 4\end{array}$ \\
\hline $\begin{array}{c}\text { Aislar sílabas } \\
\text { y fonemas al } \\
\text { inicio de una } \\
\text { palabra }\end{array}$ & .007 & $5(0-8)$ & $5(0-8)$ & $3(0-7)$ & $3(0-8)$ & Nivel $2>$ Nivel 3 \\
\hline $\begin{array}{l}\text { Detección de } \\
\text { rimas }\end{array}$ & .000 & $7(2-11)$ & $5(0-12)$ & $5(0-10)$ & $7(4-12)$ & $\begin{array}{l}\text { Nivel } 1>\text { Nivel } 2 \\
\text { Nivel } 1>\text { Nivel } 3 \\
\text { Nivel } 3>\text { Nivel } 4\end{array}$ \\
\hline $\begin{array}{l}\text { Conteo de } \\
\text { sílabas }\end{array}$ & .000 & $13(0-14)$ & $6(0-14)$ & $5(0-14)$ & $0(0-3)$ & $\begin{array}{l}\text { Nivel } 1>\text { Nivel } 3 \\
\text { Nivel } 1>\text { Nivel } 4 \\
\text { Nivel } 2>\text { Nivel } 4\end{array}$ \\
\hline $\begin{array}{l}\text { Omisión de } \\
\text { sílabas }\end{array}$ & .000 & $3(0-4)$ & $4(0-5)$ & $0(0-5)$ & $0(0-3)$ & $\begin{array}{l}\text { Nivel } 1>\text { Nivel } 4 \\
\text { Nivel } 2>\text { Nivel } 3 \\
\text { Nivel } 2>\text { Nivel } 4\end{array}$ \\
\hline Prueba total & .000 & $41(19-62)$ & $37(12-71)$ & $24(5-59)$ & $16(9-29)$ & $\begin{array}{l}\text { Nivel } 1>\text { Nivel } 3 \\
\text { Nivel } 1>\text { Nivel } 4 \\
\text { Nivel } 2>\text { Nivel } 3 \\
\text { Nivel } 2>\text { Nivel } 4\end{array}$ \\
\hline
\end{tabular}

Es importante señalar que en todas las tareas en las que existen diferencias significativas, los grupos que obtienen menores puntuaciones son el grupo 3 y el grupo 4 , es decir, los conformados por niños monolingües rurales y niños bilingües provenientes de zonas rurales.

\section{Hipótesis 3}

Existe relación entre el nivel de desarrollo de los aspectos fonológicos, memoria verbal, aspectos morfosintácticos y semánticos del lenguaje oral y la conciencia fonológica, conocimiento alfabético y demás habilidades prelectoras en los niños de la muestra.

Tabla 3. Correlaciones bivariadas entre las habilidades lingüísticas y las habilidades prelectoras

\begin{tabular}{cccccccccc}
\hline & & $\begin{array}{c}\text { Conocimiento } \\
\text { alfabético }\end{array}$ & $\begin{array}{c}\text { Reconocer } \\
\text { palabras }\end{array}$ & $\begin{array}{c}\text { Contar } \\
\text { palabras }\end{array}$ & $\begin{array}{c}\text { Aislar sílabas } \\
\text { y fonemas al } \\
\text { inicio de la } \\
\text { palabra }\end{array}$ & Rimas & $\begin{array}{c}\text { Contar } \\
\text { sílabas }\end{array}$ & $\begin{array}{c}\text { Omitir } \\
\text { sílabas }\end{array}$ \\
\hline $\begin{array}{c}\text { Discriminación } \\
\text { auditiva de } \\
\text { fonemas }\end{array}$ & rho &, $347^{* *}$ &, $238^{*}$ &, $261^{*}$ &, $316^{* *}$ & .023 & .092 &, $217^{*}$ \\
\cline { 2 - 9 } & Sig. & .001 & .022 & .012 & .002 & .828 & .382 & .037 \\
\hline
\end{tabular}


Ricardo Canales G.; Esther Velarde C.; Magali Meléndez J.; Susana Lingán H.

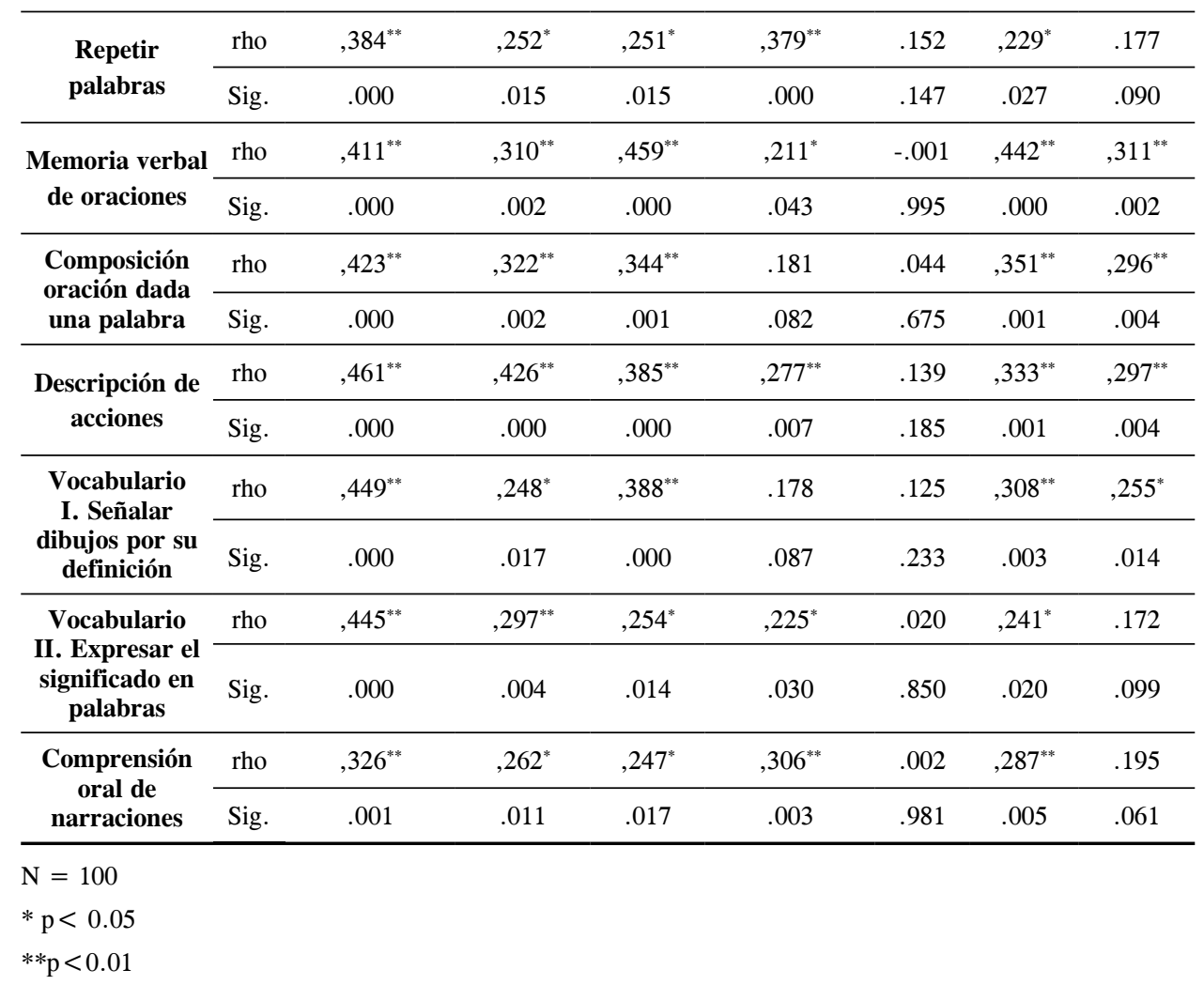

En la tabla 3 se aprecia la correlación entre las habilidades lingüísticas y las habilidades prelectoras. Los resultados demuestran que existe correlación significativa entre ambas variables en la mayoría de sus indicadores. Así tenemos, que la discriminación auditiva de fonemas correlaciona de forma moderada con el reconocimiento alfabético. De igual forma, la repetición de palabras asociada a los aspectos fonológicos se asocia significativamente con el conocimiento alfabético y la tarea de aislar sílabas y fonemas al inicio de las palabras. De otro lado, la memoria verbal correlaciona de forma moderada con todas las habilidades prelectoras, a excepción de la tarea de rimas. La tarea de señalar el dibujo de acuerdo a su significado se asocia de forma moderada con el conocimiento alfabético y la tarea de contar palabras. Asimismo, la tarea de expresar el significado de las palabras se asocia fuertemente con el conocimiento alfabético. Por último, la comprensión oral de narraciones se asocia moderadamente con las tareas de conocimiento alfabético y aislar sílabas y fonemas al inicio de las palabras.

Vemos entonces que las habilidades prelectoras que se asocian de manera más consistente con las habilidades lingüísticas son el conocimiento alfabético y el aislamiento de sílabas y fonemas al inicio de las palabras. Mientras que la habilidad que no se relaciona con las habilidades lingüísticas es la detección de rimas. 


\section{DISCUSIÓN}

Los estudios sobre la posible influencia de las condiciones socioeconómicas y factores socioculturales en el desarrollo psicológico de los niños en el Perú son escasos. A los estudios pioneros de González (1995) sobre la psicología del niño peruano y los trabajos sobre formación de conceptos en niños bilingües y monolingües en Ayacucho (2006), Problemas psicológicos en la comunicación lingüística en el Perú (op. cit.), "Exploración del desarrollo del lenguaje en el niño peruano menor de 3 años: un modelo interactivo", se aúnan en los últimos tiempos los trabajos de Reátegui, N., 1990: "Estructuras cognitivas y afectivas de madres y niños andinos" (Unicef - Ministerio de Planeamiento, La Paz, Bolivia), y en el 2008: "Niños rurales y andinos: condiciones de aprendizaje y desarrollo cognitivo afectivo", donde se impulsa un punto de vista interdisciplinario para el estudio de algunos de los grandes problemas del país, tal como es el tema de la pobreza, la marginalidad y el desarrollo cognitivo en nuestros niños.

Asimismo, en los trabajos de Canales (2012) se explora el tema de las habilidades psicolingüísticas, bilingüismo y el aprendizaje de la lectura en zonas urbanas y rurales de Lima y del interior del pais (Huancavelica), encontrando que de todos modos el desarrollo de las habilidades prelectoras se ve afectada por la condición del bilingüismo, en particular del bilingüismo incipiente.

Lo que se ha encontrado en el estudio actual es que los niños de 4 a 6 años que estudian en instituciones educativas estatales de las áreas urbanas y rurales, subdivididos en grupos por niveles de marginalidad, yendo del nivel mínimo -zona urbana y monolingüe castellano- hasta el nivel máximo -zona rural y bilingüe quechua- castellano-, se ven afectados en el desarrollo del lenguaje oral, obteniendo las menores puntuaciones el grupo de nivel 4, es decir, el conformado por niños bilingües provenientes de zonas rurales (Quintaojos, Huancavelica). Existen diferencias significativas en discriminación auditiva de fonemas, memoria verbal de oraciones, composición de oraciones, descripción de acciones, vocabulario I, comprensión oral de narraciones. Un resultado complementario respecto al lenguaje oral es el hecho de que no existan diferencias significativas en dicha variable entre los grupos de nivel 1, Lima-Cercado, y de nivel 2, Vitarte-Huaycán.

De otro lado en relación con las habilidades prelectoras, en particular la conciencia fonológica, también se hallaron diferencias significativas a nivel de discriminación auditiva de fonemas, memoria verbal de oraciones, composición de oraciones, descripción de acciones, vocabulario I, comprensión oral de narraciones. No fue discriminativa la tarea de rimas.

En este resultado principal, veríamos la directa relación que existe entre la condición de marginalidad y los factores disortogénicos (al interior de ello, el bilingüismo) con el desarrollo del lenguaje oral y la formación de las habilidades prelectoras. De acuerdo a ello, los niños que viven en condiciones de marginalidad socioeconómica: pobreza extrema, propia de las zonas rurales, aunado a la situación de diglosia (coexistencia de dos lenguas en la comunidad), se verán afectados en su desarrollo lingüístico oral, es decir en los componentes fonológicos, morfológicos, léxicos, sintácticos, semánticos y pragmáticos. 
Al respecto, hay que indicar que en la literatura se viene discutiendo el tema del bilingüismo y su repercusión en los procesos de aprendizaje. En estudios realizados hasta la década del 60 , se planteaba que si el niño aprende la segunda lengua tempranamente no presentaría mayor dificultad porque progresivamente irá diferenciando el uso y manejo de ambas de acuerdo al contexto. En estudios posteriores (Lanza, 1992; Reich, 1986), se apreció que los niños que aprendían la segunda lengua posterior a los tres años -cuando ya han conformado su aparato psicolingüístico materno-, no tenían mayores dificultades en el desarrollo del resto de sus capacidades cognitivas. El punto crítico al respecto es que en el Perú los niños no estructuran bien dicho aparato psicolingüístico por factores sociales, culturales y de interacción madre-niño. En el estudio de González (op. cit.), se aprecia que dichas interaciones lingüísticas, base para el desarrollo lingüístico del niño, son pobres en cantidad y calidad en zonas rurales. Cuando a las madres campesinas les preguntaban por qué no le hablaban a sus niños de pecho, respondían: "para qué le voy a hablar, si no me entiende". La mayor complicación se presenta cuando dichos niños asisten a la escuela (educación inicial) y son atendidos educativamente en lengua española. De ahí es que muchas veces el resultado no es la condición de bilingüe, sino de semilingüe.

Algo similar ocurre con la conciencia fonológica y las otras habilidades prelectoras. Parece ser claro, entonces, que los factores disortogénicos estarían afectando el proceso de estructuración del sistema psicolingüístico mental en función de la lengua materna, lo cual luego tendrá repercusiones en el aprendizaje de la lectura y la escritura. No ocurriría dicho fenómeno con los niños monolingües-castellano de la capital.

De otro lado, las correlaciones halladas entre diversas habilidades lingüísticas y las prelectoras nos estarían indicando, en términos generales, la directa relación entre el lenguaje oral y las habilidades prelectoras, lo cual a su vez es el soporte del aprendizaje de la lectura.

Este resultado debe llevarnos a reflexionar acerca de las deficiencias en el lenguaje oral y en la conciencia fonológica que presentan nuestros niños que viven en condiciones de marginalidad, socioeconómica y cultural-lingüística, y, a partir de ahí, empezar a entender por qué nuestros alumnos aparecen en los últimos lugares del mundo en las evaluaciones internacionales sobre lectura (PISA 2009, PISA 2012).

\section{CONCLUSIONES}

1. Se hallaron diferencias significativas en el conjunto de las puntuaciones en los niños de la muestra diferenciados por niveles de marginalidad, en desarrollo del lenguaje oral y en habilidades prelectoras: conocimiento alfabético, reconocimiento de palabras, conteo de palabras, aislar sílabas y fonemas, conteo de sílabas, omisión de sílabas. En dicha evaluación los niños de marginalidad de nivel 4, zona rural y bilingüe, aparecían consistentemente con los menores puntajes. 
2. Se halló relación entre las habilidades lingüísticas -aspectos fonológicos, memoria verbal, aspectos morfosintácticos y semánticos- y las habilidades prelectoras -conocimiento alfabético, reconocimiento de palabras, conteo de palabras, aislar sílabas y fonemas, conteo de sílabas, omisión de sílabas-.

3. No se encontraron diferencias significativas en el desarrollo del lenguaje oral entre los niños de marginalidad de nivel 1 y los de nivel 2 (ambos grupos de Lima Metropolitana).

4. Se comprobó la validez de los instrumentos utilizados para examinar las variables: condición lingüística, lenguaje oral y habilidades prelectoras.

5. A base de los resultados hallados, los especialistas del área, psicólogos, educadores, psicopedagogos, así como también las autoridades -direcciones de educación, Gobiernos regionales, etc.-, podrán contar con una base de conocimientos confiables que le permitan diseñar programas psicoeducativos de intervención para mejorar el desarrollo del lenguaje oral y las habilidades prelectoras, especialmente con los niños en mayor situación de marginalidad, los de zonas rurales y de condición bilingüe (en este caso, quechua-castellano).

\section{REFERENCIAS BIBLIOGRÁFICAS}

Berko,J. y Berstein, N. (1999): Psicolingüística. Madrid: Mc. Graw-Hill e Interamericana.

Bruner, J. (1986): El habla del niño. Aprendiendo a usar el lenguaje. Buenos Aires: Edit Paidos.

Canales, R. y Velarde, E. (2006). El valor de la palabra. Vida y obra de Raúl Gonzáles Moreyra. Lima. Edic. E. Velarde.

Canales, R. (2012) Diferencias en procesos y habilidades psicolingüisticas en niños monolingües castellano y bilingües quechua- castellano. Estudio de procesos psicolinguísticos y lectura en niños de $1^{\circ}$ grado. Lima. Instituto de Investigaciones Psicológicas. UNMSM (En Prensa).

Chomsky, N. (1979): Reseña de CONDUCTA VERBAL de B.F. Skinnner. En: Nudler, O. $(1,979)$. Problemas epistemológicos de la psicología. México. Edit. Trillas.

González, R. (2006) - La formacion de conceptos en niños bilingües. En. Problemas psicolingüísticos en el Perú. Lima. Edic. N. Reátegui.

- Problemas psicológicos en la comunicación lingüística en el Perú. En. Problemas psicolingúsiticos en el Perú. Lima. Edic. N. Reátegui.

- Exploración del desarrollo del lenguaje en el niño peruano menor de 3 años: un modelo interactivo.

González, R. (1995): Psicología del niño peruano. Lima: Universidad de Lima. Fac. de Psicología.

González, R. (2008) Test breve de bilingüismo quechua- español TBB. En: Instrumentos psicolingúsiticos. Lima. Edic. N. Reátegui. 
González, Raúl (1,993): Bilingüismo y Problemas de Aprendizaje. Revista de Problemas de Aprendizaje .C.E.P. Palestra Año 3 Nº 1 - 1993.

Pozzi-Escot, I. (1984) El castellano como segunda lengua en el Perú. Lima. Cielo Abierto

Ramos Sánchez, J. L. et al: Prueba para la evaluación del lenguaje oral. ELO. Madrid. EOS

Reátegui, N. (2008): Niños rurales y andinos: condiciones de aprendizaje y desarrollo

cognitivo afectivo. Lima: Foro Educativo.

Sellés, P. et al (2008): Batería de inicio a la lectura para niños de 3 a 6 años. BIL 3-6". Madrid. ICCE.

Velarde C. Esther.(2001). Relación entre la conciencia fonológica y el nivel de decodificación y comprensión lectora en niños de 8 años del 3er grado de primaria de dos niveles socio-económicos del Cercado del Callao. Tesis de Maestría en Educación. Lima. UNIFE.

Velarde C. Esther.(2009). Elaboración y aplicación de un programa metafonológico en niños (as) de 8 a 10 años de $3^{\circ}$ y $4^{\circ}$ grado de primaria del cercado del Callao". Tesis Doctoral en Educación. UNMSM. Lima. ANR.

Vigotsky Lev, S: Pensamiento y Lenguaje. Edit. Lautaro. Buenos Aires. 1964. 